\title{
Unilateral Salpingo-oophorectomy
}

National Cancer Institute

\section{Source}

National Cancer Institute. Unilateral Salpingo-oophorectomy. NCI Thesaurus. Code C94469.

The surgical removal of either the left or right ovary and its corresponding fallopian tube. 